\title{
Mehr Licht! La belle mort des classiques
}

Mehr Licht! Der natürliche Tod der Klassiker

More light! The natural death of the classics

\section{Frédéric Weinmann}

\section{OpenEdition}

\section{Journals}

Édition électronique

URL : http://journals.openedition.org/ceg/5568

DOI : $10.4000 /$ ceg.5568

ISSN : 2605-8359

Éditeur

Presses Universitaires de Provence

Édition imprimée

Date de publication : 1 octobre 2013

Pagination : 13-32

ISSN : 0751-4239

\section{Référence électronique}

Frédéric Weinmann, "Mehr Licht! La belle mort des classiques », Cahiers d'Études Germaniques [En ligne], 65 | 2013, mis en ligne le 01 octobre 2019, consulté le 24 novembre 2020. URL : http:// journals.openedition.org/ceg/5568; DOI : https://doi.org/10.4000/ceg.5568 


\title{
Mehr Licht! \\ La belle mort des classiques \\ Frédéric WEINMANN \\ Lycée Hélène Boucher, Paris
}

\begin{abstract}
« Il faut vous imaginer le jeune Gauck, à treize ans, en train de lire son Egmont le soir dans son lit... Si, si, autrefois, je lisais de toute façon beaucoup de Schiller, autrefois ${ }^{1}$. »
\end{abstract}

"Qu'est-ce qu'un classique? », se demande Charles-Augustin SainteBeuve dans sa Causerie du lundi du 21 octobre 1850. Après un bref rappel historique d'où se dégagent deux critères déterminants - d'une part la consécration au fil du temps, d'autre part un fond de richesse littéraire « qui a suite et consistance, qui fait ensemble et tradition, qui se compose, se transmet et qui dure »-, le grand critique littéraire reçu à l'Académie française cinq ans plus tôt par son ami Victor Hugo signale qu'au tout début du XIX ${ }^{\mathrm{e}}$ siècle, et plus précisément sous l'Empire, "l'idée de classique, chez quelques esprits résistants et encore plus chagrins que sévères, se resserra et se rétrécit étrangement ». À l'appui de ses affirmations, il compare la définition du concept dans le premier Dictionnaire de l'Académie (1694) à celle de la sixième édition (1835), où l'accent est mis sur les notions de « modèles » à imiter et de « règles » à suivre : « cette définition, écrit-il, a été faite évidemment par les respectables académiciens nos devanciers en présence et en vue de ce qu'on appelait alors le romantique, c'est-à-dire en vue de l'ennemi ». La nouvelle génération, à laquelle il appartient, a désormais conquis le pouvoir intellectuel et entend faire une place à ses propres chefs d'œuvre. C'est pourquoi Sainte-Beuve suggère de « renoncer à ces définitions restrictives et craintives » avant de proposer une quatrième définition, la définition moderne, la sienne :

\footnotetext{
1 Le président de la République Joachim GAUCK cité d'après Der Spiegel $\mathrm{n}^{\circ} 23,4$ juin 2012, p. 45 : « Sie müssen sich den 13-jährigen Gauck vorstellen, wie er abends im Bett seinen Egmont las... Ja, ja, ich habe damals überhaupt viel Schiller gelesen, damals. »
} 


\begin{abstract}
Un vrai classique, comme j'aimerais à l'entendre définir, c'est un auteur qui a enrichi l'esprit humain, qui en a réellement augmenté le trésor, qui lui a fait faire un pas de plus, qui a découvert quelque vérité morale non équivoque, ou ressaisi quelque passion éternelle dans ce cœur où tout semblait connu et exploré ; qui a rendu sa pensée, son observation et son invention, sous une forme n'importe laquelle, mais large et grande, fine et sensée, saine et belle en soi; qui a parlé à tous dans un style à lui et qui se trouve aussi celui de tout le monde, dans un style nouveau sans néologisme, nouveau et antique, aisément contemporain de tous les âges. ${ }^{2}$
\end{abstract}

Cette définition que Sainte-Beuve lui-même qualifie de "grandiose et flottante ou, pour tout dire, généreuse " libère les auteurs classiques de l'esthétique du même nom, c'est-à-dire des notions de régularité, sagesse, modération, raison, nuance, élégance, netteté, justesse, bon sens. Elle permet de ranger sous ce terme "Homère avant tout, le père du monde classique", Dante et Milton, " la grande figure de Shakespeare », " le Corneille de Polyeucte, de Cinna et d'Horace », Molière et La Fontaine "bien avant Boileau, même avant Racine », bref d'intégrer «les vrais et souverains génies », « ces autorités primitives, auxquelles on revient tôt ou tard dans les jours d'émancipation ». Le document est particulièrement précieux car il permet de dater au jour près la remise en cause des valeurs classiques en France au milieu du XIX ${ }^{\mathrm{e}}$ siècle. Ce n'est pas n'importe qui, mais un des académiciens les plus en vue qui estime que « le Temple du goût $[\ldots]$ est à refaire » pour qu'il devienne "le Panthéon de tous les nobles humains». Rétrospectivement, certains des noms évoqués «depuis le plus libre des génies créateurs et le plus grand des classiques sans le savoir, Shakespeare, jusqu'au tout dernier des classiques en diminutif, Andrieux » ne manquent pas de surprendre. Malgré les vœux de Sainte-Beuve, "les Addison, les Pellisson, les Vauvenargues » n'ont pas intégré le nouveau Panthéon littéraire. Cependant, le détail importe moins que le principe. L'essentiel est que les fondements du classique changent de manière radicale autour de 1850.

La nouvelle conception renvoie de manière explicite à Goethe, cité par trois fois dans cette seule Causerie, même si ce n'est pas encore - il faut le souligner - à titre d'auteur classique, mais en tant que " roi de la critique». Sainte-Beuve ne commente pas simplement l'une des références préférées du Weimarien, à savoir le verset XIV.2 de L'Évangile selon saint Jean (« Il y a plus d'une demeure dans la maison de mon père »), pour insister sur « la libre diversité des génies ». Il reprend surtout, dans une traduction personnelle, le fameux jugement rapporté par Eckermann en date du 2 avril 1829 :

J'appelle le classique le sain, et le romantique le malade. Pour moi le poëme des Niebelungen est classique comme Homère; tous deux sont bien portants et vigoureux. Les ouvrages du jour ne sont pas romantiques parce qu'ils sont nouveaux, mais parce qu'ils sont faibles, maladifs ou malades. Les ouvrages

\footnotetext{
${ }^{2}$ Charles-Augustin Sainte-Beuve, Causeries du lundi, Paris, Garnier, 1851, t. III, p. 30-
} 43 , ici p. 33. 
anciens ne sont pas classiques parce qu'ils sont vieux, mais parce qu'ils sont énergiques, frais et dispos. Si nous considérons le romantique et le classique sous ces deux points de vue, nous serions bientôt tous d'accord. ${ }^{3}$

La référence au prince des poètes n'a rien de surprenant à une époque où l'élite intellectuelle française se montre particulièrement à l'écoute de l'Allemagne. Elle permet de maintenir et, à la fois, de dépasser la dualité classique / romantique qui s'était cristallisée dans les années 1820. Comme l'a très bien montré Stéphane Zékian dans L'Invention des classiques, le canon établi au XVIII ${ }^{\mathrm{e}}$ siècle avait survécu à la Révolution. La prépondérance des auteurs du Grand Siècle ne s'était pas démentie ; après une brève période d'hésitation, des lectures parfois opposées avaient repris et justifié les mêmes références obligées. Au lendemain de la Restauration encore, les ultras s'étaient réapproprié assez vite la référence au Roi soleil, malgré son instrumentalisation par l'Empire, et opposaient la gloire du XVII siècle à la décadence d'un XVIII ${ }^{\mathrm{e}}$ siècle réduit aux Lumières, c'est-à-dire à la Terreur, tandis que les libéraux défendaient de leur côté «l'hypothèse militante d'un siècle de deux cent ans ${ }^{4} »$. Les débats littéraires opposaient moins défenseurs et détracteurs du ou des classique(s) que différentes lectures de cet héritage.

La donne se modifia brusquement avec la parution d'une série d'articles dans la Paris Monthly Review, repris en 1823 sous le titre fameux de Racine et Shakespeare. Choqué par l'accueil que les jeunes libéraux avaient réservé à une troupe anglaise au théâtre de la Porte Saint-Martin, Stendhal avait pris position non pas pour une quelconque doctrine classique, mais contre toute doctrine classique. Dans la deuxième édition (1825), il ajoute même un troisième chapitre dans lequel il parvient, par une étonnante pirouette, à faire de Racine un romantique :

Le romanticisme est l'art de présenter aux peuples les œuvres littéraires qui, dans l'état actuel de leurs habitudes et de leurs croyances, sont susceptibles de leur donner le plus de plaisir possible.

Le classicisme, au contraire, leur présente la littérature qui donnait le plus grand plaisir possible à leurs arrière-grands-pères.

Sophocle et Euripide furent éminemment romantiques [...]. Imiter aujourd'hui Sophocle et Euripide, et prétendre que ces imitations ne feront pas bâiller le Français du dix-neuvième siècle, c'est du classicisme.

Je n'hésite pas à avancer que Racine a été romantique ; il a donné, aux marquis de la cour de Louis XIV, une peinture des passions, tempérée de l'extrême dignité qui alors était de mode, et qui faisait qu'un duc de 1670, même dans les épanchements les plus tendres de l'amour paternel, ne manquait jamais d'appeler son fils Monsieur. [...]

Il faut du courage pour être romantique, car il faut hasarder.

3 Ibid., p. 36. Cette traduction diffère de la traduction « officielle» publiée par Délerot en 1861 avec une introduction de Sainte-Beuve.

${ }^{4}$ Stéphane ZÉKIAN, L'invention des classiques, Paris, CNRS éditions, 2012, p. 96. 
Le classique prudent au contraire, ne s'avance jamais sans être soutenu, en cachette, par quelque vers d'Homère, ou par une remarque philosophique de Cicéron, dans son traité De Senectute.

Point n'est besoin de retracer ici les aléas de la bataille romantique ni de multiplier les citations. Le texte précurseur de Stendhal suffit à montrer comment, en 1823, la notion de classique s'oppose tout à coup à celle de romantique et se réduit pour ainsi dire à l'idée de règle et d'imitation. Il illustre également les contorsions auxquelles les partisans du romantisme doivent se livrer pour épargner leurs critiques à quelques écrivains du canon officiel. Comme Stendhal faisant de Racine un romantique, Hugo imaginera dans la préface de Cromwell (1827) les scènes que l'auteur d'Esther et d'Athalie aurait écrites «s'il n'eût pas été paralysé comme il l'était par les préjugés de son siècle, s'il eût été moins souvent touché par la torpille classique $^{6} »$. Racine n'est pour rien, d'après lui, dans la doctrine qui se serait formée au tournant des $\mathrm{XVIII}^{\mathrm{e}}$ et $\mathrm{XIX}^{\mathrm{e}}$ siècles " comme une pénultième ramification du vieux tronc classique, ou mieux comme une de ces excroissances, un de ces polypes que développe la décrépitude et qui sont bien plus un signe de décomposition qu'une preuve de vie »: Delille « est le père (lui, et non Racine, grand Dieu !) d'une prétendue école d'élégance et de bon goût qui a flori récemment ${ }^{7} »$.

C'est pour échapper à ces contorsions que Sainte-Beuve propose une définition moins restrictive que les Jeune-France à l'époque d'Hernani ou les académiciens de 1835. La bataille romantique est terminée, le temps de la synthèse est arrivé. Ce geste conçu comme généreux marque moins une réconciliation des points de vue que la consécration et la victoire définitive du Cénacle. La Monarchie de Juillet constitue le laboratoire d'un nouveau canon littéraire qui s'épanouira pleinement dans la deuxième moitié du siècle. L'exemple de Molière illustre à merveille ce phénomène. Hugo, Musset, Vigny ainsi encore que George Sand (dans son drame de 1851) esquissent une nouvelle image du grand auteur classique : à l'instar d'Alceste, d'Arnolphe ou d'Harpagon, le dramaturge se transforme sous leurs plumes en un personnage sombre, pathétique, mélancolique, souffrant des infidélités de sa femme et suscitant la pitié. Michelet, à la même époque, explique son œuvre à partir des conditions politiques dans la France de Louis XIV. Molière devient alors - Ralph Albanese l'a montré il y a déjà bien longtemps ${ }^{8}$ - un porte-parole de la bourgeoisie victorieuse. Nisard, Saint-Marc Girardin ou

${ }^{5}$ StendHal, Racine et Shakespeare, Paris, Bossange / Delaunay / Mongie, 1823, p. 43-45.

${ }^{6}$ Victor HugO, Cromwell, Paris, Ambroise Dupont, 1828, p. XXXIV.

${ }^{7}$ Ibid., p. XLII-XLIII.

${ }^{8}$ Ralph Albanese, "The Moliere Myth in Nineteenth-Century France", in Robert L. Mitchell (dir.), Pre-text, Text, Context. Essays on Nineteenth-Century French Literature, Columbus, Ohio State University Press, 1980, 239-254, notamment p. 241 : « However, it is undoubtedly the scholarly criticism of Nisard and Saint-Marc Girardin, as well as that of SainteBeuve, that, between 1830 and 1848, provided the greatest impetus to the development of Moliérism. » 
Brunetière se servent de son œuvre pour rejeter les innovations de leur temps et combattre la décadence qu'ils croient constater. En insistant sur la nature éternelle de l'homme, ils le mettent au service d'une morale et d'une idéologie susceptibles de dépasser les clivages sociaux. Dans son article « Was macht den Klassiker zum Klassiker? », Andrea Grewe a résumé l'instrumentalisation de Molière en ces termes:

Cette interprétation de Molière connaît son apogée sous la Troisième République. Après le traumatisme de la défaite qui fit prendre conscience de la nécessité d'une régénération nationale globale, la tendance moralisante, la tendance nationaliste et la tendance bourgeoise de l'interprétation de Molière ont convergé : l'étude des classiques et des valeurs qu'ils préservent sont conçus comme le moyen approprié pour surmonter les conséquences de cet effondrement du fait qu'on voit en eux la source d'une « saine » morale bourgeoise, du patriotisme et d'un héritage commun susceptible de dépasser les clivages sociaux et idéologiques.

L'essentiel du canon élaboré au XVIII ${ }^{\mathrm{e}}$ siècle a survécu à la profonde remise en cause des valeurs et des principes mêmes du jugement esthétique à l'époque romantique. Ce ne sont pas tant les noms qui ont changé que la raison de leur présence au palmarès. Les auteurs classiques n'y figurent plus désormais comme les représentants supérieurs d'une esthétique éternelle, mais - pour reprendre une expression du Moliériste en 1882 - comme «la plus parfaite incarnation du génie français ${ }^{10} »$. La continuité apparente ne doit donc pas faire illusion et nous tromper sur la signification profonde de cette mutation : dans la deuxième moitié du $\mathrm{XIX}^{\mathrm{e}}$ siècle, les classiques deviennent des porte-parole de la nation.

Malgré d'importantes différences en surface, la littérature allemande connaît le même processus à la même époque. De fait, la parution (entre 1835 et 1842) de la monumentale Geschichte der poetischen National-Literatur der Deutschen de Georg Gottfried Gervinus ouvre une ère nouvelle pour la définition du et des classique(s) dans les territoires de langue allemande. Même si cet historien libéral n'emploie nulle part le terme (qui, selon René Wellek, ne s'impose de manière générale qu'en $1887^{11}$ ), il est à l'origine d'une nouvelle vision de la littérature allemande : dans son esprit, les dix

9 Andrea GREWE, « Was macht den Klassiker zum Klassiker? », in Wilhelm VOßKAMP (dir.), Klassik im Vergleich. Normativität und Historizität europäischer Klassiken. DFGSymposion 1990, Stuttgart / Weimar, Metzler, 1993, 242-258, p. 245-246 : "Ihren Höhenpunkt erlebt diese Auslegung Molières in der Dritten Republik. Unter dem Schock der Niederlage und im Bewusstsein der Notwendigkeit einer umfassenden nationalen Erneuerung konvergieren die moralisierende, die nationalistische und die bürgerliche Tendenz der Moliere-Interpretation: Das Studium der Klassiker und der in ihnen aufbewahrten Werte wird als das geeignete Mittel erkannt, um die Folgen des Zusammenbruchs zu überwinden, da in ihnen die Quelle einer „gesunden“ bürgerlichen Moral, des Patriotismus und eines die sozialen und ideologischen Gräben überwindenden gemeinsamen Erbes gesehen wird. »

10 «L'hérésie de M. Scherer», Le Moliériste n 37, avril 1882, p. 39.

11 Voir René WeLlEK, Grenzziehungen. Beiträge zur Literaturkritik, Berlin / Köln / Mainz, Kohlhammer, 1972, p. 61. 
années de production de Goethe et Schiller à Weimar en forment le sommet indépassable. Tous leurs prédécesseurs sont considérés comme de simples devanciers tandis que leurs successeurs, romantiques inclus, contribuent fatalement au déclin des lettres germaniques. Dès l'introduction au premier volume, il esquisse un récit dans lequel l'histoire de la littérature allemande forme un flux continu et doté de sens ; il distingue quatre périodes qui s'enchaînent de manière graduée. Les valeurs établies du XVIII ${ }^{\mathrm{e}}$ siècle sont rabaissées ; à côté de Garve, Gellert, Engel, Möser, Pfeffel, ce sont notamment Klopstock et Wieland qui souffrent de cette redéfinition du canon.

La clé de voûte de sa construction est l'invention d'une symbiose entre Schiller et Goethe. Même si Wilhelm von Humboldt avait stipulé très tôt une parenté spirituelle entre les deux écrivains, la conception dominante tendait jusque-là à les opposer, à les concevoir comme un couple antithétique. Schiller (qui jouissait alors d'un plus grand renom) apparaissait comme l'auteur engagé, prenant fait et cause pour la libération nationale; Goethe comme le porte-parole de l'art pur, indifférent au temps présent. Gervinus part d'ailleurs de ce schéma établi ; dans Über den Göthischen Briefwechsel (1836), il se prononce encore en faveur de Schiller. Mais dans le cinquième volume de son histoire de la poésie nationale allemande (1842), il finit par résoudre l'opposition en minimisant les différences et en introduisant un troisième terme. Selon lui, les deux poètes représenteraient à eux seuls la totalité des possibilités artistiques et humaines :

Et ainsi, les lignes de cette créature bicéphale se croisent en eux de si multiples
façons que ce n'est en quelque sorte que sous cette forme entrelacée qu'ils nous
présentent un tout commun qu'il s'agit pour nous de goûter et d'exploiter sans
les séparer, comme c'était dans l'intention des deux hommes eux-mêmes.

Ces phrases, explique Karl Robert Mandelkow, constituent en quelque sorte « le manifeste fondateur d'une notion de classique essentielle pour 1'histoire de la réception ${ }^{13}$ ». Peter Uwe Hohendahl retrace quant à lui en détail l'influence considérable de cet ouvrage dans les décennies suivantes ${ }^{14}$ : même les historiens favorables au romantisme ou à Wieland et Jean-Paul, par exemple, reprennent en fin de compte le schéma et les catégories proposés par Gervinus. Ainsi Julian Schmidt ajoute-t-il en 1855 un supplément à sa Geschichte der deutschen Literatur im neunzehnten Jahrhundert dans lequel

12 Georg Gottfried Gervinus, Geschichte der poetischen National-Literatur der Deutschen. Fünfter Band, Leipzig, Engelmann, $1853^{4}$, p. 475 : « Und so durchkreuzen sich die Linien des doppelseitigen Wesens in Beiden so vielfach, daß sie uns gleichsam erst in dieser verschlungenen Gestalt ein gemeinsames Ganzes darstellen, an dem wir uns ungetrennt freuen und aufbauen sollen, wie es in der Absicht der Männer selber lag. »

13 Karl Robert MandelKow, Goethe in Deutschland. Rezeptionsgeschichte eines Klassikers, München, Beck, 1980, t. I, p. 123 : " das Gründungsmanifest eines wirkungsgeschichtlichen Klassikbegriffs ».

${ }_{14}$ Voir Peter Uwe HoHENDAHL, Literarische Kultur im Zeitalter des Liberalismus 18301870, München, Beck, 1985, p. 162-166. 
il avance, quoiqu'il ne partage pas le point de vue pessimiste de Gervinus sur la littérature allemande d'après 1806 :

Tout le monde est d'accord pour dire que nous avons eu en Allemagne un âge classique, qui commence à un moment déterminé et finit à un moment déterminé ; mais on se pose rarement la question de savoir combien de temps il a duré à proprement parler. [...] On a hérité du passé une certaine conception des écrivains qu'on appelle nos classiques. Parmi eux, on compte entre autres Klopstock, Wieland et Herder. Mais si l'on entend par le terme d'œuvre classique une œuvre qui livre à la postérité le contenu riche et important d'une grande époque dans sa forme parfaite, on pourra difficilement qualifier de classique aucune des œuvres de ces trois écrivains. Les belles-lettres allemandes n'acquièrent conséquence et cohérence qu'au moment où Goethe et Schiller s'unissent; et cela cesse à la mort de Schiller. L'union des deux poètes datant de 1794, cela dure donc exactement onze ans ; voilà la période que nous devons appeler notre période classique, c'est-à-dire le moment où les esprits supérieurs de la nation entretiennent l'un avec l'autre une nécessaire relation intime, où leurs écrits renferment la plus haute expression de la culture allemande et où la forme reçoit toute la perfection dont la langue allemande est absolument capable. $^{15}$

Après l'échec de la révolution de 1848 , le discours critique se fait moins passionné que pendant le Vormärz. À l'heure où la philologie institutionnelle se met en place, la critique littéraire commence à se dépolitiser, à réclamer de nouveau l'autonomie de l'art et à se cantonner aux jugements de valeur littéraires. Mais les principaux publicistes de l'époque (Gustav Freytag, Rudolf Gottschall, Karl Gutzkow, Hermann Marggraff, Robert Prutz et Julian Schmidt) ont débuté leur carrière avant la révolution et continuent pour l'essentiel sur la voie tracée par Gervinus. À partir de 1850, l'union sacrée de Schiller et Goethe relève par conséquent d'une évidence que personne ne remet plus en question : l'inauguration à Weimar, le 4 septembre 1857, de la double statue représentant les deux amis d'une part et de la statue solitaire de Wieland d'autre part consacre un état de fait. Malgré la vaine protestation de Karl Gutzkow dans Nur Schiller und Goethe? (1859), Wieland et Klopstock

15 Julian SCHMIDT, Weimar und Jena in den Jahren 1794-1806, Leipzig, Herbig, 1855 (2., durchaus umgearbeitete Auflage), t. I, p. 1-2: « Daß wir in Deutschland ein classisches Zeitalter der Literatur gehabt, mit einem bestimmten Anfange und einem bestimmten Ende, darüber ist alle Welt einig; selten aber legt man sich die Frage vor, wie lange es eigentlich gewährt habe. [...] Man hat aus alter Zeit die Vorstellung von gewissen Schriftstellern, die man unsere Classiker nennt. Dazu rechnet man unter andern Klopstock, Wieland und Herder. Wenn man aber unter einem classischen Werk dasjenige versteht, welches in einer vollendeten Form den reichen und bedeutenden Inhalt einer Culturepoche der Nachwelt überliefert, so wird man kaum eins der Werke dieser drei Schriftsteller ein classisches nennen. Folge und Zusammenhang tritt in die schöne deutsche Literatur erst ein, als Goethe und Schiller sich verbinden; sie hört auf mit Schiller's Tod. Das Bündnis der beiden Dichter fällt ins Jahr 1794, es dauerte also gerade elf Jahre; und diesen Zeitraum müssen wir als unsern classischen bezeichnen, d.h. als denjenigen, in welchem die hervorragenden Geister der Nation in einer innern nothwendigen Beziehung zu einander stehen, in dem ihre Schriften den höchsten Ausdruck der deutschen Bildung enthalten und in dem die Form diejenige Vollendung erhält, die der deutschen Sprache überhaupt möglich ist. " 
ne sont plus désormais pour l'institution littéraire que des figures marginales et il n'y a pas encore de place pour Jean-Paul ou pour Kleist. Même Gottschall, qui conteste pourtant l'absolue suprématie de Schiller et Goethe ainsi que le déclin de la littérature allemande après 1806, stipule l'existence du classicisme de Weimar. Dans l'introduction à sa Deutsche Nationalliteratur in der ersten Hälfte des neunzehnten Jahrhunderts (1855), il écrit au début de la première partie, intitulée "Die Classiker », que Wieland et Klopstock ne méritent pas ce qualificatif car « seuls quelques fils les relient encore à l'époque présente ${ }^{16}{ } \gg$. Il s'agit déjà ici, pour reprendre les analyses de Hohendahl, de légitimer une tradition nationale susceptible de concurrencer celle des autres nations :

La « légende du classicisme » ne devient compréhensible qu'au vu d'une configuration historique spécifique : le nationalisme allemand en pleine croissance recherchait une identité culturelle. Le libéralisme primitif la trouva dans la poésie allemande et l'établit historiquement grâce à la catégorie âge classique. $^{17}$

Dans les années 1860 , les manuels scolaires, longtemps fidèles au canon issu du XVIII ${ }^{\mathrm{e}}$ siècle et en retard par rapport aux réflexions contemporaines, intègrent le schéma issu de Gervinus. Simultanément, les historiens contestent l'opposition entre classique et romantique qui dominait la discussion littéraire depuis les années 1830. Dans la préface à sa Deutsche Nationalliteratur (1855), Gottschall reprenait toujours le schéma traditionnel :

Les classiques créèrent notre forme artistique sur le modèle antique et avec un esprit humaniste ; les romantiques détruisirent à nouveau cette forme pour émanciper l'imagination de toute tradition et rendre la poésie populaire $[\ldots] .{ }^{18}$

Dix ans plus tard, cette opposition se résorbe. Dans son essai consacré à Novalis (1865), le jeune Wilhelm Dilthey se dresse contre l'opinion dominante en rendant hommage à l'une des figures centrales du premier romantisme. En 1867, dans son discours inaugural à l'université de Bâle, il va plus loin encore et esquisse une synthèse qui se distingue de celle de ses prédécesseurs en ce qu'elle associe le romantisme aux grandes heures de la littérature allemande. Grâce à lui, Lessing se détache des Lumières et devient

\footnotetext{
${ }^{16}$ Rudolf GotTSCHALl, Deutsche Nationalliteratur in der ersten Hälfte des neunzehnten Jahrhunderts, Breslau, Trewendt, $1861\left(1855^{1}\right)$, p. 3 : «Von Klopstock und Wieland führen nur noch wenige Fäden in unsere Gegenwart hinüber. »

${ }^{17}$ HOHENDAHL, Literarische Kultur, p. 162 : « Die „Klassiklegende“ wird erst verständlich vor dem Hintergrund einer spezifischen historischen Konstellation: Der wachsende deutsche Nationalismus suchte nach einer kulturellen Identität. Der Frühliberalismus fand sie in der deutschen Dichtung und fixierte sie historisch durch die Kategorie eines klassischen Zeitalters. $)$

${ }^{18}$ GotTSChall, Deutsche Nationalliteratur, p. XII : "Die Classiker schufen uns die künstlerische Form nach antikem Vorbild und mit humanem Geiste; die Romantiker zerstörten diese Form wieder, um die Phantasie von gegebenen Traditionen zu emancipiren und die Dichtung volksthümlich zu machen [...].»
} 
le père fondateur d'un mouvement intellectuel qui permet l'éclosion à la fois du classicisme et du romantisme. De cette manière, Dilthey présente la culture prussienne comme la condition nécessaire à l'épanouissement culturel de l'Allemagne et prépare le terrain aux lectures nationales :

\footnotetext{
De fait, le pouvoir qui se fait constamment sentir dans l'évolution de ce mouvement tient au besoin, fondé d'un point de vue historique, de fonder une conception de la vie et du monde dans laquelle l'esprit allemand trouve satisfaction. ${ }^{19}$
}

Dilthey influence donc moins le canon littéraire par l'ajout de nouveaux noms que par la redéfinition des rapports entre l'Aufklärung et le classicisme d'une part, entre Weimar et le romantisme d'autre part. L'intégration du romantisme au canon littéraire se voit confirmée dans l'ouvrage de Rudolf Haym Romantische Schule, paru en 1870. Pour l'auteur, le romantisme n'est plus la négation, mais la continuation logique du classicisme. L'un n'exclut plus l'autre ; les deux mouvements concourent à la gloire de la littérature nationale. Cette reconnaissance des romantiques, enfin débarrassés de l'image de réactionnaires catholiques qu'ils devaient pour une bonne part à Heine, sur ce point l'allié de Goethe ${ }^{20}$, n'empêche pas au même moment la sacralisation du prince des poètes.

L'une des premières manifestations du culte qui lui sera rendu pendant tout le deuxième Reich se trouve dans les conférences que Herman Grimm donna à l'université de Berlin en 1874-1875 : l'Olympien y prend place aux côtés de Dante et de Shakespeare. Sa neutralité politique, qui constituait un défaut aux yeux de la critique libérale, se mue au contraire en une qualité dans la rhétorique de l'Allemagne bismarckienne : « Le travail de Goethe a aidé à créer le sol sur lequel, aujourd'hui, nous semons et récoltons. Il fait partie des plus distingués fondateurs de la liberté allemande ${ }^{21}$. $\gg$ Comme dans la troisième République de l'autre côté du Rhin, les classiques constituent ici les porte-parole de la nation. Cette fonction a été bien perçue par Hohendahl :
Autour de 1870, les personnes cultivées étaient d'accord sur le fait que les Allemands possédaient, comme leurs voisins européens, un corpus d'auteurs et d'œuvres classiques grâce auquel on pouvait légitimement se définir comme un « grand peuple». Quelques soient les changements et les déplacements opérés

\footnotetext{
19 Wilhelm Dilthey, « Die dichterische und philosophische Bewegung in Deutschland 1770-1800. Antrittsvorlesung in Basel 1867 ", in Gesammelte Schriften, Göttingen, Vandenhoeck \& Ruprecht, $1990^{8}$, t. 5 (Die geistige Welt), p. 13 : « Und zwar lag die stetig fortwirkende Macht im Verlauf dieser Bewegung in dem geschichtlich begründeten Drang, eine Lebens- und Weltansicht zu begründen, in welcher der deutsche Geist seine Befriedigung finde. $\gg$

${ }^{20}$ Voir Karl Robert MANDELKOW, "Heinrich Heine und die deutsche Klassik », in Orpheus und Maschine. Acht literaturgeschichtliche Arbeiten, Heidelberg, Stiehm (Poesie und Wissenschaft IX), 1976, 63-85.

${ }^{21}$ Herman GRIMM, Goethe. Vorlesungen gehalten an der Kgl. Universität zu Berlin, Berlin, Hertz, 1877, t. 1, p. 9 : « Goethe's Arbeit hat den Boden schaffen helfen, auf dem wir heute säen und ärnten. Er gehört zu den vornehmsten Gründern der Deutschen Freiheit. »
} 
après 1870 (réévaluation de Hölderlin et de Kleist, découverte de Büchner), le processus d'élaboration de ce canon est pour l'essentiel achevé. 22

L'examen des Histoires de la littérature étrangère, telles qu'on en publiait à l'époque ${ }^{23}$, suggère en effet que, par un accord tacite, les grandes nations modernes se reconnaissent mutuellement une période de gloire et de domination intellectuelle. Chacune a désormais son poète immortel (Dante, Cervantès, Shakespeare, Molière ou Racine, Goethe), suivi d'une cohorte d'écrivains moins importants avec lesquels il fonde l'âge d'or d'une littérature et incarne l'esprit de la nation. Le socle classique, dans lequel l'esthétique classique (der Klassizismus) ne joue en fin de compte aucun rôle, n'échappe pas à quelques aménagements ultérieurs : les romantiques allemands s'établissent une fois pour toutes dans les années 1890, en particulier grâce à Hermann Bahr, Ricarda Huch et les symbolistes français. Les naturalistes, notamment Gerhardt Hauptmann, amorcent la canonisation de Georg Büchner; et le cercle de Stefan George découvre Hölderlin peu avant que les expressionnistes imposent Heinrich von Kleist. Mais même s'il faut attendre encore longtemps pour voir Heinrich Heine sur un pied d'égalité avec les classiques de Weimar (l'est-il d'ailleurs vraiment ?), la liste des grands auteurs de la littérature allemande est établie à la fin du XIX ${ }^{\mathrm{e}}$ siècle, c'est-à-dire en 1914. Et elle s'articule autour du pivot que représente le classicisme de Weimar.

Certes, les protestations ne manquent pas contre le culte rendu aux classiques. Dans la première des Considérations intemporelles (1873), Friedrich Nietzsche s'en prend à l'ouvrage de David Friedrich Strauß, Der alte und der neue Glaube (1872), dans lequel Lessing est présenté comme le précurseur de Goethe et de Schiller. Nietzsche dénonce les conséquences désastreuses de ce mythe national où les grands écrivains sont réduits à des figures de cire, de simples jetons du commentaire savant, un sujet de discussion pour le thé, le bien d'un public s'imaginant avoir vaincu la culture française par la guerre de 1870. Nietzsche voit en Lessing l'agent de destruction du classicisme européen que ni Goethe ni Schiller n'étaient en mesure de rétablir. Sous la République de Weimar également, les classiques subissent les assauts de virulents ennemis. Dans son manifeste Die bürgerliche Literaturgeschichte und das Proletariat (1922), Max HermannNeiße rejette " la vénération inconditionnelle des idoles de l'art posés en

\footnotetext{
${ }^{22}$ HOHENDAHL, Literarische Kultur, p. 220 : « Es bestand um 1870 unter den Gebildeten Einigkeit darüber, daß die Deutschen wie ihre europäischen Nachbarn ein Korpus klassischer Autoren und Werke besaßen, durch das man sich als „Kulturvolk“ legitimieren konnte. Der Prozeß der Ausbildung dieses Kanons ist bei allen Änderungen und Verschiebungen, die sich nach 1870 vollzogen (Aufwertung Hölderlins und Kleists, Entdeckung Büchners) im Wesentlichen abgeschlossen. »

23 Voir Frédéric WEINMANN, «Remise en cause du canon dans les Histoires de la littérature étrangère », Revue d'histoire littéraire de la France (à paraître 2014).
} 
classiques par l'évaluation bourgeoise ${ }^{24}$ ». En 1929, le critique théâtral Herbert Jhering constate quant à lui la «mort des classiques ${ }^{25}$ » et Bertold Brecht l'approuve :

S'ils sont morts, quand sont-ils morts ? La vérité est qu'ils sont morts à la guerre. Ils font partie de nos victimes. S'il est vrai qu'au moment de s'en aller à la guerre, les soldats avaient Faust dans leur sac à dos, ceux qui en revinrent ne 1'avait plus. ${ }^{26}$

La remise en cause de l'héritage classique se fera plus forte encore au lendemain de la Seconde Guerre mondiale. C'est ainsi que Richard Alewyn déclare en 1949 : «Entre nous et Weimar, il y a Buchenwald ${ }^{27}$.» De telles provocations, réactions exacerbées au culte inconditionnel du Bildungsbürgertum, ne sauraient masquer que les classiques ont survécu à plusieurs guerres, même s'il fallut pour cela une régulière adaptation du discours critique à de nouveaux contextes sociopolitiques. Grâce aux travaux de Karl Robert Mandelkow ${ }^{28}$, les dates clés de cette constante relecture sont bien connues : après la glorification de Goethe comme précurseur de l'unification dans le Reich bismarckien, la République de Weimar en fit le garant de la démocratie contre l'esprit prussien. Les nazis, au contraire, célébrèrent à travers lui la gloire germanique. Après 1945, il incarna dans la République d'Adenauer une tradition intellectuelle propre à l'Occident, un point d'ancrage, un fondement apolitique de l'identité nationale, tandis qu'à l'Est, l'héritage classique permettait de résister à toutes les tentatives et tentations de modernité - du moins jusqu'à l'époque d'Honecker, jusqu'à la (re)découverte d'E.T.A Hoffmann, de Jean Paul ou de Kleist, jusqu'à ce que Christa Wolf écrive dans l'introduction de son ouvrage consacré à Karoline von Günderrode :

${ }^{24}$ Max HerRmanN-Neiße, « Die bürgerliche Literaturgeschichte und das Proletariat », Die Aktion, 1922, cité d'après Walter Fähnders / Martin Rector (dir.), Literatur im Klassenkampf. Zur proletarisch-revolutionären Literaturtheorie 1919-1923, München, Hanser, 1971, p. 71 : " die unbedingte Verehrung der künstlerischen Götzen, die von der bürgerlichen Wertung als Klassiker aufgestellt wurden ».

${ }^{25}$ Herbert JeHRing, Reinhardt, Jessner, Piscator oder Klassikertod, Berlin, Rowohlt, 1929.

${ }^{26}$ Berthold BRECHT, « Gespräch über Klassiker » (etwa 1929, fragmentarisch), in Schriften zum Theater I. 1918-1933, Frankfurt/Main, Suhrkamp, 1963, p. 146-156, ici p. 146 : «Wenn sie gestorben sind, wann sind sie gestorben? Die Wahrheit ist: Sie sind im Krieg gestorben. Sie gehören unter unsere Kriegsopfer. Wenn es wahr ist, daß Soldaten, die in den Krieg zogen, den Faust im Tornister hatten, die aus dem Krieg zurückkehrten, hatten ihn nicht mehr. » Voir aussi dans le même volume « Wie soll man heute Klassiker spielen? » (25. Dezember 1926), p. 89-91.

27 Richard ALEWYN, " Goethe als Alibi ? ", Hamburger Akademische Rundschau 3, 1948/1949, vol. 8-10, p. 686 : «Zwischen uns und Weimar liegt Buchenwald. »

28 Voir en particulier Karl Robert MANDELKOW, « Wandlungen des Klassikbildes in Deutschland im Lichte gegenwärtiger Klassikkritik (1977) » et « West-östliche Goethe-Bilder. Zur Klassikrezeption im geteilten Deutschland (1982) », in Gesammelte Aufsätze und Vorträge zur Klassik- und Romantikrezeption in Deutschland, Frankfurt am Main, Peter Lang, 2001, 21 40 et 41-60. 
FRÉDÉRIC WEINMANN

L'histoire littéraire des Allemands, aux mains des professeurs de lycée et des professeurs d'université, axée sur la peinture monumentale de ses classiques, s'est débarrassée avec insouciance et inconscience des personnages catalogués comme « imparfaits », y compris dans le passé le plus récent, y compris dans le verdict lourd de conséquences que Georg Lukács a prononcé contre Kleist et contre les romantiques.

En R.F.A. également, une profonde remise en cause de l'image et de l'interprétation des classiques s'est amorcée avec la biographie « sacrilège » de Goethe par Richard Friedenthal en 1963, le congrès des germanistes à Munich en octobre $1966^{30}$ et la discussion animée qui suivit le discours d'Emil Staiger «Literatur und Öffentlichkeit » à Zürich en décembre de la même année, ou la publication des actes du Second Wisconsin Workshop sous le titre « Die Klassik-Legende » en 1971. À partir de ce moment-là, une nouvelle génération s'efforce de désacraliser et de replacer les écrivains classiques dans une perspective historique :

Fini donc ce geste pieux, de droite comme de gauche, vénérant et par là momifiant absolument tout ce qui touche audit classicisme de la cour de Weimar. Le temps est enfin venu de considérer à nouveau l'élément classique comme quelque chose d'historique et de vivant plutôt que quelque chose d'éternel et de mort. Car c'est la seule façon de détruire le caractère normatif qui a si longtemps adhéré à ce modèle. ${ }^{31}$

Un excellent témoignage de leur entreprise de déconstruction est le recueil Die Geschichtlichkeit der deutschen Klassik dans lequel Walter Müller-Seidel rassemble plusieurs articles sur la littérature allemande autour de 1800 et s'en prend à la vénération a-historique des grandes figures de l'art :

La pensée construite sur les catégories de l'intemporalité trouve son expression dans la notion d'homme classique ; et par là, il ne s'agit pas de penser l'époque du classicisme et le classicisme de Goethe uniquement comme une phase de sa création littéraire, mais comme quelque chose de bien supérieur, qui vise des

29 Karoline von Günderrode, Der Schatten eines Traumes. Gedichte, Prosa, Briefe, Zeugnisse von Zeitgenossen. Hg. und mit einem Essay von Christa Wolf, Berlin, Luchterhand, 1981, p. 5-6 : « Die Literaturgeschichte der Deutschen, in den Händen von Studienräten und Professoren, orientiert an den Kolossalgemälden ihrer Klassiker, hat sich leichtherzig und leichtsinnig der als „unvollendet“ abgestempelten Figuren entledigt, bis in die jüngste Zeit, bis zu dem folgenreichen Verdikt, das Georg Lukács gegen Kleist, gegen die Romantiker aussprach. »

${ }^{30}$ Benno VON WIESE / Rudolf HENß (dir.), Nationalismus in Germanistik und Dichtung. Dokumentation des Germanistentages in München vom 17-22 Oktober 1966, Berlin, Erich Schmidt, 1967.

31 Reinhold Grimm / Jost Hermand (dir.), Die Klassik-Legende. Second Wisconsin Workshop, Frankfurt am Main, Athenäum, 1971, p. 14 : «Weg also mit jener frommen Geste von rechts und links, die alles und jedes an dieser Weimarer Hofklassik verehrt und damit mumifiziert. Es ist endlich an die Zeit, das Klassische wieder als etwas Historisch-Lebendiges und nicht als etwas Überzeitlich-Totes zu betrachten. Denn nur so läßt sich jener normative Charakter zerstören, der diesem Leitbild so lange angeheftet hat. » 
formes d'existence éternelles. Le classique et le moderne se fondent dans un flou étonnant, comme si l'on avait affaire à des notions interchangeables. ${ }^{32}$

De manière plus discrète, moins dramatique, presque nostalgique, un phénomène similaire se produit en France sous le signe de la mémoire nationale. La trace la plus vivace de ce questionnement demeure Les lieux de mémoire, une énorme publication collective née d'un séminaire que Pierre Nora tint à l'École des Hautes Études en Sciences Sociales de 1978 à 1981. Il s'agissait, face à « la disparition rapide de notre mémoire nationale ${ }^{33}$ ", d'entreprendre un inventaire des valeurs fondatrices : les fêtes, les emblèmes, les institutions, les dictionnaires, les musées... Les deux volumes consacrés à la nation, en particulier, mettaient en évidence le caractère artificiel des valeurs considérées comme proprement françaises, les « objets les plus représentatifs de sa tradition, que nous reconnaissons toujours comme nôtre, mais que nous ne pouvons plus vivre comme telle ». S'il jugeait qu'il était encore trop tôt pour entreprendre " l'analyse d'ordre philosophique qui, de l'extérieur du fait national, et passant derrière son décor, nous expliquerait ce mystère en pleine lumière », Pierre Nora prenait acte du malaise suscité par ces valeurs et, en compagnie de ses coauteurs, réintroduisait l'histoire dans des notions qui passaient jusque-là pour éternelles.

Le chapitre consacré par Daniel Milo aux classiques scolaires retraçait, en la comparant au cas anglo-saxon, l'élaboration du canon littéraire français depuis le Rapport de la Commission des livres classiques, déposé début 1803, jusqu'aux listes d'auteurs cités pour la préparation du bac français à l'aube des années 1980. Cette étude, pleine de tableaux et de statistiques, permet de suivre les fluctuations du corpus scolaire sur presque deux siècles. Elle rappelle que, malgré d'évidentes convergences, "les deux "classiques", scolaire et culturel, ne sont pas équivalents ». Elle suggère enfin que «le canon scolaire a vécu » :

C'est donc une entité hybride qu'est la mémoire culturelle de la quasi-totalité des Français. Elle mêle Molière et Paul Kenny, Boileau et Jean Bruce, comme le montre le tableau VIII. Ce tableau est l'expression d'une anomalie culturelle qui n'est pas sans rappeler la tension qui régnait sous l'Ancien Régime entre les classiques antiques et les cultures nationales. Le $\mathrm{XIX}^{\mathrm{e}}$ siècle a résolu cette tension grâce surtout au mouvement profond de démocratisation qui s'est traduit par une expansion scolaire sans précédent. Quoi, donc, de plus naturel que la

32 Walter MÜLLER-SEIDEL, Die Geschichtlichkeit der deutschen Klassik. Literatur und Denkformen um 1800, Stuttgart, Metzler, 1983, p. 16 : «Das Denken in den Kategorien der Zeitlosigkeit findet im Begriff des klassischen Menschen seinen Ausdruck; und damit ist nicht nur an die Zeit der Klassik und an Goethes Klassik als eine Phase seines literarischen Schaffens zu denken, sondern an etwas weit mehr, das auf überzeitliche Daseinsformen zielt. Auf eine merkwürdig undeutliche Art gehen das Klassische und das Moderne ineinander über, als hätte man es mit auswechselbaren Begriffen zu tun. »

33 Pierre NORA, «Présentation », in Pierre NORA (dir.), Les lieux de mémoire, Gallimard, Quarto, 1997, t. I, p. 18 pour l'ensemble des citations de ce paragraphe. 
nouvelle vague « démocratique », celles des années 1960, ait à son tour fait éclater le canon des classiques nationaux ? $^{34}$

Cet article cherche donc moins à déterminer les auteurs classiques et les critères du classique qu'à sonder la fonction d'un tel canon, son origine et son influence. Quelques années plus tard, une équipe du Centre de Recherche sur l'Institution Littéraire (créé à Paris III en 1989) approfondit le sujet et présenta ses travaux dans le numéro 19 de la revue Littératures classiques sous le titre «Qu'est-ce qu'un classique ? » (automne 1993). Dans un article intermédiaire paru sous le même titre dans le Bulletin des Bibliothèques de France, Alain Viala résumait les principaux résultats de l'enquête et ramenait la question du classique à un phénomène de réception. La valeur classique d'une œuvre ne serait pas une valeur en soi, mais une valeur relative : il s'agit selon lui d'un bien symbolique, d'une valeur d'échange permettant d'être reconnu, accepté, récompensé. L'étiquette «classique » désigne une valeur sûre, stable, susceptible de résister aux crises des systèmes politiques, économiques et idéologiques. Si elle implique certaines propriétés formelles, ce serait uniquement la pluralité des destinataires possibles, la logique de la «multiple alliance » :

Le classique, on l'a vu, ce n'est donc pas la valeur forcément la plus élevée, mais
celle qui peut le mieux circuler, c'est la valeur d'échange sûre. [...] Il nous
semble donc hasardeux, pour le moins, de regarder le classicisme comme une
esthétique qui transcenderait les contingences de lieu et de temps et dont des
œuvres diverses seraient des manifestations correspondant entre elles par-delà
leurs différences historiques; ou de le regarder comme la caractéristique
exceptionnelle d'une époque historique exceptionnelle. Le classicisme peut se
définir comme un processus qui saisit des œuvres pour les faire entrer dans une
même logique de communication et de socialisation ; il les saisirait plus ou
moins fortement en fonction des propriétés qu'elle offrent à cet égard. ${ }^{35}$

Une telle analyse semble devoir mettre fin une fois pour toutes au mythe $\mathrm{du}$ et des classique(s). Sans même prendre la peine de commenter l'opposition désuète entre classique et romantique, elle conteste à la fois l'idée de supériorité absolue et celle d'esthétique atemporelle. En d'autres termes, elle réduit le classique à une pure fonctionnalité et traite le concept comme un phénomène historique. Dans les années suivantes, Viala poursuit son œuvre salutaire au sein du G.R.I.H.L. (Groupe de Recherche Interdisciplinaire sur l'Histoire du Littéraire) et publie en 2002 un ouvrage collectif sous le titre éloquent de "Quelques "XVII" siècle" : Fabrications, usages et réemplois ${ }^{36} »$. Dans un article consacré notamment à la critique de Sainte-Beuve par Proust, Branka Sarancic, par exemple, montre que le

34 Daniel Milo, « Les classiques scolaires », in Pierre NORA (dir.), Les lieux de mémoire, t. II, p. 2085-2130, ici p. 2122.

${ }_{35}$ Alain ViaLA, «Qu'est-ce qu'un classique », BBF, 1992/1, p. 6-15.

${ }^{36}$ Cahiers $d u$ Centre de recherches historiques, $\mathrm{n}^{\circ} 28-29,2002$. Disponible en ligne à http://ccrh.revues.org/812. 
« nettoyage » entrepris par les tenants de la modernité littéraire autour de 1900 n'aboutit en fin de compte qu'à réactualiser le mythe :

\begin{abstract}
Dans le contexte de politisation accrue du champ intellectuel, qui exacerbe les conflits inhérents à sa structure intérieure, il ne s'agit plus de défendre une certaine « idée de la nation », mais le principe même de l'autonomie du littéraire. Face au renforcement des tensions idéologiques, provoqué par l'affaire Dreyfus, il n'est plus question de mettre la littérature au service d'une «cause supérieure », mais de l'ériger en force subversive qui s'opposera à toute tentative d'instrumentalisation de la pensée. ${ }^{37}$
\end{abstract}

Au début du $\mathrm{XXI}^{\mathrm{e}}$ siècle, on peut donc tenir pour acquis que le $\mathrm{XVII}^{\mathrm{e}}$ siècle français (tout comme le "foyer des muses » weimarien dans les pays de langue allemande) a été instrumentalisé depuis le début du XIX ${ }^{\mathrm{e}}$ siècle, en particulier dans le but de définir des littératures nationales. La question en suspens consiste à savoir dans quelle mesure la déconstruction du mythe échappe à son objet ou reste au contraire fidèle à la tradition. En 2001, Marie Bury et Georges Forestier organisèrent à la Sorbonne un colloque sur les "Jeux et enjeux des théâtres classiques" au $\mathrm{XIX}^{\mathrm{e}}$ et au $\mathrm{XX}^{\mathrm{e}}$ siècle de manière à interroger l'usage que les romantiques et leurs adversaires firent des notions de classique et de néo-classique. Dans la présentation des actes, ils observaient que la dimension polémique de ces deux notions, héritée du premier XIX ${ }^{\mathrm{e}}$ siècle, demeure « toujours vivace en tant que repoussoir »:

[...] il se trouve encore aujourd'hui des spécialistes de théâtre qui, après Stendhal, Hugo et Barthes, combattent un Racine étroitement classicisé, et qui épousent radicalement des points de vue partisans liés à l'évolution propre à la théorie littéraire et inscrits dans un contexte politique précis. ${ }^{38}$

Conscients de la pérennité du schéma, ils remarquaient en outre que les auteurs dramatiques survivent mieux que les autres écrivains classiques dans la mesure où leurs œuvres, par le biais de la mise en scène, sont inévitablement soumises à une réactivation. Cependant, on peut s'étonner de les voir ensuite reprendre à leur compte la définition des auteurs classiques donnée par Lanson ( une possibilité permanente de pensées et de sentiments $\left.{ }^{39} \gg\right)$ et donc se demander si les discussions animées sur la notion de classique ne conduisent pas dans certains cas à la revivifier : dans ce cadre, le classicisme français n'est plus défini comme apogée d'une culture, mais il demeure un moment particulier, un tournant décisif - où reviennent par la petite porte les chefs-d'œuvre du Siècle de Louis XIV.

Comme nous l'avons rappelé dans l'avant-propos, il est possible de se dresser contre le $\mathrm{XIX}^{\mathrm{e}}$ siècle avec des arguments du $\mathrm{XIX}^{\mathrm{e}}$ siècle. La

\footnotetext{
${ }^{37}$ Branka SARANCIC, " Le "siècle classique" à l'épreuve de la "modernité littéraire" ", Cahiers du Centre de recherches historiques, $\mathrm{n}^{\circ}$ 28-29, 2002, p. 1-30, p. 28.

${ }^{38}$ Littératures classiques $n^{\circ} 48$ (« Jeux et enjeux des théâtres classiques »), 2003, p. 9-10.

${ }^{39}$ Gustave LANSON, Hommes et livres, Études morales et littéraires, Paris, Lécène / Oudin, 1895 , p. 36
} 
contestation ou la réactualisation des auteurs et des valeurs classiques n'implique pas nécessairement un dépassement du paradigme postromantique. Ainsi, dans Le répertoire classique sur la scène contemporaine, Brigitte Prost constate que « depuis les années 1970, se sont multipliées les mises en scène des textes anciens et [que], parmi ceux-ci, les œuvres de Molière, Racine et Corneille ont occupé une place prépondérante » avant de s'interroger sur ce phénomène : "Il y a dans un tel retour de faveur, écrit-elle, un paradoxe dont l'ampleur mérite d'être interprétée. Pourquoi persister en effet à mettre en scène des classiques ? » La réponse qu'elle apporte à cette question, bien loin d'interroger les sous-entendus du panthéon littéraire, se contente d'en moderniser la rhétorique en lui découvrant de nouvelles raisons d'exister: l'intérêt toujours vivace pour les œuvres des classiques tient selon elle au « rapport particulier qu'entretiennent ces auteurs au temps, à l'histoire et à la mémoire ${ }^{40}$ ». Son avant-propos («Petites remarques sur les classiques $»)$ offre un bon exemple de perpétuation inconsciente d'un schéma issu du siècle romantique.

Les chercheurs qui, depuis quarante ans, ont éprouvé un malaise vis-à-vis des références classiques, interrogé les fondements du canon littéraire, introduit la relativité et la temporalité dans un modèle jusque-là conçu comme immuable et éternel, nous ont cependant appris à nous méfier du mythe des écrivains classiques en montrant qu'il (sur)vit depuis le milieu, voire le début $\mathrm{du} \mathrm{XIX}^{\mathrm{e}}$ siècle grâce une adaptation permanente aux nouveaux contextes sociopolitiques. Le propre de l'idéologie (au sens de George Dumézil ou de Louis Dumont) est son caractère d'évidence aux yeux de ceux qui la partagent. Hohendahl rendait bien compte de cette difficulté quand il écrivait à propos de l'héritage de Gervinus : "Cette façon de voir est devenue si évidente pour les générations postérieures qu'aujourd'hui encore, nous avons du mal à y reconnaître une construction à côté de laquelle d'autres constructions étaient également possibles ${ }^{41}$. » On est donc en droit de se demander si la remise en cause des valeurs classiques à la toute fin du $\mathrm{XX}^{\mathrm{e}}$ siècle n'est qu'un nouvel avatar de l'ancien système ou l'amorce d'une véritable révolution. Cette mutation est-elle comparable à la rupture des années 1830-1850, où un nouveau paradigme vit le jour, en France comme en Allemagne ? Ou sommes-nous toujours dépendants du système qui s'est mis en place au milieu du XIX ${ }^{\mathrm{e}}$ siècle, c'est-à-dire en bref de l'idéologie nationale?

Malgré les stéréotypes qui continuent largement de dominer l'opinion publique, on peut espérer qu'une définition vraiment nouvelle du terme « classique » est en train de voir le jour. Ainsi, dans un manuel destiné aux étudiants, Alain Genétiot prend pour point de départ les acquis encore trop

\footnotetext{
40 Brigitte PROST, Le répertoire classique sur la scène contemporaine, Rennes, PUR (Le spectaculaire), 2010, p. 17-18.

${ }^{41}$ HoHENDAHL, Literarische Kultur, p. 171 : «Späteren Generationen ist diese Sichtweise so selbstverständlich geworden, daß wir noch heute Mühe haben, sie als eine Konstruktion zu erkennen, neben der auch andere möglich waren. »
} 
confidentiels des précédentes décennies : il rappelle que, contrairement au terme de "classique», attesté au XVII ${ }^{\mathrm{e}}$ siècle dans le sens de «faisant autorité » et réservé aux seuls auteurs de l'Antiquité, « la présentation d'un classicisme homogène, incarnation d'une essence à un moment de grâce de la civilisation française, est une invention de l'histoire littéraire des Modernes et $\mathrm{du} \mathrm{XVIII}^{\mathrm{e}}$ siècle relayée par l'enseignement scolaire au $\mathrm{XIX}^{\mathrm{e}}$ siècle dans un projet de définition culturelle de l'identité nationale ${ }^{42} »$. La mauvaise réputation de la littérature du $\mathrm{XVII}^{\mathrm{e}}$ siècle tiendrait d'ailleurs au fait que l'âge postmoderne reste héritier de la période moderne et conserve une vision postromantique. Et de souligner une différence essentielle entre le baroque dont les traits distinctifs caractérisent bel et bien une époque par-delà les frontières nationales - et le classicisme qui « n'a pas cette homogénéité historique $^{43} \gg-$ certaines œuvres dites classiques relevant même de l'esthétique baroque.

La période que Genétiot désigne par le terme de classique ne se limite pas aux glorieuses décennies 1660-1680, elle s'étend de Malherbe à Fénelon. Ainsi entendu, le classicisme français correspondrait à une époque qui s'autoproclame supérieure et s'expliquerait par un contexte historique : "les institutions sociales ont organisé et fourni un système de contraintes à la création littéraire du temps ${ }^{44}$. » Il n'est plus question d'affirmer la supériorité absolue des œuvres et des artistes de ce temps, il s'agit plutôt d'étudier une configuration historique « en excluant les autres pays, non par nationalisme comme au XIX $\mathrm{X}^{\mathrm{e}}$ siècle, mais pour faire œuvre historique précise en marquant la singularité de cette littérature par rapport à ses propres modèles et sources d'inspiration en fonction de la spécificité socio-historique du cadre original de la centralisation absolutiste ${ }^{45} \gg$.

Plus récemment encore, Jean-Claude Vuillemin attaque de front le concept de " classicisme » dans un effort de "resémantisation » du baroque. Se réclamant de Michel Foucault, il conteste néanmoins la notion d'épistémè classique que ce dernier définissait par la combinaison de la grammaire générale, de l'analyse des richesses et de l'histoire naturelle, et lui préfère une épistémè baroque "que Foucault a profondément méconnue à cause vraisemblablement des œillères de l'histoire littéraire et culturelle de son temps ${ }^{46} »$. Selon Vuillemin, l'émergence de la science moderne à l'époque de Galilée, Mersenne, Hobbes, Gassendi, Descartes, Roberval, Boulliau fournit à la pensée de nouveaux cadres de références et permet la naissance du sujet moderne : en passant du géocentrisme à l'héliocentrisme, l'homme baroque abandonne le théocentrisme au profit d'un anthropocentrisme qui le rend rebelle à toute idée de vérité absolue et universelle. Pour étayer sa conception

${ }^{42}$ Alain GENÉTIOT, Le classicisme, Paris, PUF (Quadrige. Manuels), 2005, p. 2.

${ }^{43}$ Ibid., p. 3.

${ }^{44}$ Ibid., p. 4.

${ }^{45}$ Ibid., p. 2-3

${ }^{46}$ Jean-Claude Vuillemin, Épistémè baroque. Le mot et la chose. Paris, Hermann (Savoir Lettres), 2013, p. 67. 
du baroque, Vuillemin retrace la « genèse du mythe classique » et insiste sur sa pérennité jusqu'à l'époque actuelle :

En fait, au lieu de l'éradication d'une notion pourtant unanimement reconnue équivoque, il y a eu solidification et légitimation de son hégémonie conceptuelle. Il en va du classicisme comme de l'histoire littéraire : c'est un mal, mais un mal nécessaire. Dans sa très large majorité la critique universitaire a validé et disséminé le point de vue d'Henri Peyre ou celui de Paul Valéry qui, après avoir lui aussi affirmé que les appellations de «classique » et de « romantique » sont « incompatibles avec la précision de la pensée, parfaitement inutiles à son développement », ajoutait qu' « il n'est pas mauvais que des termes de ce genre existent («Classique et Romantique », p. 115). ${ }^{47}$

Conscient d'un tel danger, Vuillemin rejette au contraire la "prétendue » rationalité classique qui ne représente pour lui tout au plus qu'une «des modalités ingénieuses, pour ne pas dire géniales, que l'épistémè baroque invente afin de pallier la crise profonde qu'elle traverse ${ }^{48} »$. Il refuse d'isoler le cas français du contexte général de l'Europe du XVII ${ }^{\mathrm{e}}$ siècle et s'inscrit en faux contre l'illusoire évidence du découpage "classique ». Dans ces conditions, le terme, s'il garde un sens, ne désigne plus le caractère éternel d'œuvres supérieures produites à une époque exceptionnelle de l'histoire nationale, mais tout au plus un des «multiples avatars » de la sensibilité baroque. Cette prise de conscience équivaut à son tour à une rupture épistémologique :

\footnotetext{
[...] ce fameux classicisme empreigne si fortement, et depuis si longtemps, notre vision $\mathrm{du} \mathrm{XVII}{ }^{\mathrm{e}}$ siècle que la familiarité que nous entretenons avec lui tient souvent lieu de certificat d'authenticité et de pertinence conceptuelle. Cette période, que l'on caractérise en général par une concentration étonnante de chefs-d'œuvre littéraires et par une aspiration à la perfection antique alliant régularité et mondanité, ne doit pourtant sa cohérence posthume qu'à l'oubli de multiples débats théoriques et autres âpres prises de position antagonistes qui, dans tous les domaines ou presque, l'ont vigoureusement secouée et qui, par conséquent, ne peuvent que ruiner la validité d'un marqueur esthétique hégémonique. ${ }^{49}$
}

On assiste donc bien à un tournant dans la définition du et des classiques. Dans son acception de représentants majeurs d'une culture nationale, le terme pourra bientôt être considéré comme vieilli. Les dictionnaires devraient en revanche enregistrer le sens jusqu'alors inconnu d'œuvres ou d'artistes instrumentalisés à des fins idéologiques en dépit des particularités de leur esthétique. Ce changement ouvre les portes d'une nouvelle histoire de la littérature, de la musique et des arts en général et devrait permettre de réhabiliter des talents injustement écrasés par le rouleau compresseur du paradigme postromantique. Elle devrait un jour permettre d'apprécier sans $a$

\footnotetext{
${ }^{47}$ Ibid., p. 95.

48 Ibid., p. 22.

49 Ibid., p. 40.
} 
priori les œuvres de grands auteurs du passé enfin débarrassés d'une étiquette qu'ils n'auraient pas songé à se coller eux-mêmes, ainsi que le rappelle Goethe à l'apogée de ce qu'on appellera plus tard le « classicisme de Weimar » :

Nous sommes persuadés qu'aucun auteur allemand ne se tient lui-même pour classique, et que les exigences de tout un chacun envers soi-même sont plus strictes que les prétentions confuses d'un Thersite s'élevant contre une vénérable compagnie qui ne réclame nullement qu'on admire ses efforts de manière inconditionnelle, mais qui est en droit d'attendre qu'on sache les apprécier. ${ }^{50}$

50 Johann Wolfgang von GoETHE, „Literarischer Sanscülottismus“ (1795), in Werke. Hamburger Ausgabe in 14 Bände, München, DTV, 1981, t. XII, p. 240 : «Wir sind überzeugt, daß kein deutscher Autor sich selbst für klassisch hält, und daß die Forderungen eines jeden an sich selbst, strenger sind, als die verworrnen Prätensionen eines Thersiten, der gegen eine ehrwürdige Gesellschaft aufsteht, die keineswegs verlangt, daß man ihre Bemühungen unbedingt bewundere; die aber erwarten kann, daß man sie zu schätzen wisse. » 